\title{
PROYECTOS DE RECREACIÓN Y TIC A TRAVÉS DE WWW. BIOTOPO.COM
}

\author{
(RECREATION PROYECTS AND TIC TROUGH WWW.BIOTOPO.COM)
}

Julián José Martínez Bañuelos

IES Atenea de Alcalá de Henares (España)

\section{RESUMEN}

Una de las maneras de trabajar con TIC en el aula es la de generar entidades ficticias que utilicen, tal y como ocurre en el ámbito real, estas ya no tan nuevas tecnologías. El ejemplo desarrollado durante el curso 2004/05 ha sido becado por El Corte Inglés y ha obtenido uno de los premios del concurso Investiga a través del entorno y exponlo.

Palabras clave: tecnologías de la información y comunicación, investigación acción, ecología, proyectos de recreación, diversificación curricular, comunidad virtual, biotopo.com.

\begin{abstract}
One of the ways to work with TIC in the classroom is the one to so generate organizations fictitious that use, and as it happens in the real scope. The example developed during course 2004/05 has been granted a scholarship by El Corte Inglés and has obtained one of the prizes of the aid Investigates through environment and expose it.
\end{abstract}

Key words: ecology, action-research, recreation proyect, virtual comunity, ICT, diversification program.

Pretendemos investigar y a la vez formar a nuestros alumnos a través de la recreación ficticia de una empresa: TOXICODIVER ATENEA, que tendrá como objetivo la mejora del medio ambiente. Diversificará su actividad en tres campos: 
el ecoturismo, ofreciendo al cliente itinerarios de senderismo y cicloturismo, aprovechando el magnífico entorno de la ciudad de Alcalá, la regeneración de terrenos degradados, aprovechando también un ejemplo de nuestro entorno: los taludes de carretera y el reciclaje de residuos por el interés relacional y didáctico con la física y química, tecnología y biología, atendiendo en particular al tratamiento de metales pesados. Así mismo, se realizarán actividades ligadas al estudio del impacto biológico de contaminantes en los seres vivos.

Creemos que en los IES existe un déficit en cuanto a cultura empresarial, en cuanto a formación para el autoempleo y en el conocimiento de la empresa. Este trabajo nos servirá de excusa para fomentar este espíritu empresarial.

Atenderemos, por una parte, al proceso mediante el que se crea una empresa, desde la génesis de la idea, su inscripción en el Ayuntamiento, alta en Seguridad Social, etc. Recrearemos los trámites burocráticos, de gestión y constitución que son necesarios para la puesta en marcha real de dicha actividad. Nuestros alumnos visitarán empresas del sector para el estudio de la competencia y del mercado.

Por otra parte, al desarrollar esta empresa de marcado carácter ambiental, se sumergirán en los preceptos de la ecología, que nos llevan hacia un modelo de desarrollo que pueda ser asumido por nuestro planeta, que nos permita crecer económicamente pero respetando el medio en el que vivimos. Habrán de aprender a conjugar economía y ecología (oikos: casa, nomos: administración, logos: ciencia).

Mostraremos nuestros logros a través de una exposición física, realizada en el Instituto y una exposición virtual a través de una comunidad basada en la WWW, utilizando nuestra experiencia y las herramientas preparadas en nuestro portal $\underline{w w w}$. biotopo.com. El uso de las nuevas tecnologías será una constante en nuestro trabajo. La colaboración entre alumnos utilizará esta útil herramienta.

\section{ESTRUCTURA DIDÁCTICA}

\section{Objetivos del proyecto}

- Conseguir implementar y recrear la puesta en marcha de una empresa ficticia que centra su actividad en el ámbito medio-ambiental.

- Implicar activamente a los alumnos del programa de Diversificación curricular y del programa de Compensatoria para estimular su desarrollo 
y su motivación hacia los ámbitos científico-tecnológicos y la orientación profesional y para el autoempleo.

- Potenciar el aprendizaje basado en la comunicación (Young) a través de las herramientas cooperativas incorporadas al sistema php-nuke. Implementado y desarrollado en www.biotopo.com.

- Desarrollar activamente el autoaprendizaje y el aprendizaje en comunidad en un entorno colaborativo trabajando en ambientes informáticos del tipo comunidad virtual.

- Explorar materiales, instrumentos de diferente tipología, conocer sus propiedades para su utilización con fines expresivos, comunicativos y de carácter lúdico.

- Explorar el mundo de la empresa, su funcionamiento, su gestión y su puesta en marcha.

- Fortalecer y estimular el espíritu empresarial de los alumnos de Secundaria.

- Obtener y manejar información de diferentes fuentes, tratándola de forma autónoma y crítica.

\section{Orientaciones metodológicas}

Lo que hace que una acción sea educativa no es la obtención de estados finales, sino las cualidades intrínsecas que se ponen de manifiesto en la misma forma de llevar a cabo una acción (Elliot, 1988). El significado de lo que hacemos en educación tiene sentido por sí mismo en el propio proceso en el que se produce un intercambio de los alumnos con el medio educativo complejo (Martínez Bañuelos, 2002). De esta manera la realización, difusión y participación en eventos educativos como este concurso patrocinado por El Corte Inglés nos ayuda a esta forma de aprender que los investigadores denominan inducción por sumergimiento. Así, entendemos la educación como un proceso de inducción que implica el sumergimiento en las estructuras del pensamiento, tan complejas y variopintas en todos los ámbitos del conocimiento.

En este proceso, difícil de encorsetar en una didáctica tradicional, es donde las Tecnologías de la Información y la Comunicación tienen su papel más importante: aceleran el intercambio de ideas, la comparación de las mismas, el análisis crítico y en última instancia el flujo multidireccional de información.

Creemos, por lo tanto, que poseen dos funcionalidades básicas, que en resumen son: fuente de información y canal de comunicación, las que abren a Internet las 
puertas del mundo educativo (Marqués, 1999). En este sentido parecen poseer por sí mismas unos valores para la Educación (Fernández Muñoz, 1998):

- Preparar para un trabajo cada vez más versátil, en donde la destreza en su uso se hace un valor añadido. (Educación para el empleo).

- Entender la realidad que les toca vivir a nuestros alumnos, en una sociedad tecnificada. (Educación para la vida).

- Comprender el impacto de la ciencia y la tecnología en todos los aspectos de la sociedad, que requiere, además de las disciplinas tradicionales, un punto de vista más global: educación para la responsabilidad ambiental, y para el desarrollo armonioso de las relaciones intra e inter sociedades. (Educación para el mundo).

- Desarrollar el análisis crítico de tal manera que seamos capaces de entender conceptos y desarrollarnos por nosotros mismos. (Educación para el autodesarrollo).

- La educación como una actividad placentera. (Educación para el ocio).

Es un momento de paroxismo, en el que los medios de comunicación han vivificado su carácter de fenómenos de masas, en el que nuestros alumnos antes de pasar por la Escuela han asistido a una explosión constante de información que les mediatiza y les atiborra de ideas preconcebidas, inundados de Gran Hermano, Internet, también como fenómeno de masas, puede servir para acceder a la información individualizada y de múltiples facetas en la que el espectador elige qué quiere, qué ve y a dónde se dirige.

Se hace por lo tanto imprescindible la incorporación de este medio tecnológico como herramienta extraordinaria y de proyectos que de forma interdisciplinar abarquen varias materias y relacionen conceptos, aprovechando la comunicación de los mismos mediante las poderosas herramientas que nos ofrece, cada día de forma más refinada Internet y sus tecnologías. Así en nuestra página www.biotopo.com el alumno posee un diario electrónico, un correo para comunicarse con sus compañeros, chat, posibilidad de publicar redacciones de forma inmediata, posibilidad de incorporar enlaces, usar y compartir los ya existentes, etc.

El acceso a Internet, en el entorno educativo, para nuestros jóvenes estudiantes, es una necesidad y una obligación para las autoridades educativas, podemos destacar en nuestro centro la existencia de tres aulas de informática, una de ellas dotada con la última tecnología. 
También para nuestros profesores, en el caso de la Secundaria que nos ocupa, es prescriptiva la innovación como punta de lanza que abra un camino para el uso de las TIC por los jóvenes que dentro de poco serán ciudadanos adultos, universitarios, técnicos o ciudadanos insertos en nuestra sociedad. Plantear el uso de Internet como un acceso a la información de forma crítica, que suponga el cuestionamiento de las concepciones y parcelas del pensamiento humano, ha de ser objetivo prioritario.

Hemos elegido como paradigma y marco de nuestro trabajo el de la Investigación Acción (en adelante I/A) en la que se hace imprescindible una gran conversación entre grupos de profesores, entre alumnos y profesores y entre los propios alumnos, que permita volar entre la reflexión y la acción en continuas espirales. Esta forma de trabajo quizá nos permita construir un prototipo de espacio virtual en el que enganchemos nuestra acción didáctica diaria. La indagación, experimentación de nuevas estrategias, contraste de ideas, reelaboración del currículo en un marco teórico adecuado reforzarán nuestra profesionalización.

Trabajamos en el sentido desarrollado por Kurt Lewin, el último fin de nuestro trabajo es propiciar el uso de las TIC entre los profesores de Secundaria, contribuir en la medida de las posibilidades a que el uso de éstas se realice de una forma integrada en el currículo y girando alrededor de otras actividades, para que ejerza por lo tanto su función como medio de comunicación.

Las características que diferencian a la I/A de otras metodologías de investigación (Colás, 1998) son: carácter situacional, participativa, autoevaluativa, los beneficiarios son la comunidad implicada, caracterizada por la acción reflexión, se produce la aplicación inmediata de hallazgos, la investigación se entiende como un proceso de creación sistemática de conocimientos.

\begin{tabular}{ll}
\hline \multicolumn{2}{c}{ Características diferenciales en el marco de la I/A } \\
\hline Carácter situacional & $\begin{array}{l}\text { Intentamos resolver y paliar varios problemas del } \\
\text { contexto, el aprendizaje integrado de economía y } \\
\text { ecología. }\end{array}$ \\
\hline Participativa & $\begin{array}{l}\text { Los miembros toman parte en la elaboración de } \\
\text { materiales para la web. }\end{array}$ \\
\hline Autoevaluativa & $\begin{array}{l}\text { A través delas herramientas de colaboración virtuales } \\
\text { se colabora en la evaluación de las actividades. }\end{array}$ \\
\hline Los beneficiarios son la comunidad & $\begin{array}{l}\text { El crecimiento y mantenimiento de Biotopo.com } \\
\text { redunda en la propia comunidad educativa. }\end{array}$ \\
\hline
\end{tabular}


Acción-Reflexión

Aplicación inmediata de hallazgos
El trabajo de acción en el aula, la búsqueda de recursos ecológicos, datos y diseño de nuestra empresa, etc. se convierten en fuente de datos y en objeto de reflexión.

Los productos conseguidos en la propia investigación en el aula son inmediatamente puestos a disposición de los miembros del proceso a través de enlaces ubicados en la web.

Como filosofía de aprendizaje creemos en el constructivismo en el sentido introducido por el filósofo Giambattista Vico, y desarrollado John Dewey entre otros, que pensaba que los humanos pueden sólo aprender aquello que ellos mismos construyen. Entendemos que la educación depende de la acción, el conocimiento y las ideas emergen solamente en aquellas situaciones en la que el actor es el propio alumno (Dewey, 1966).

\section{TAREAS DE LOS ALUMNOS}

En esquema, se han realizado las siguientes tareas:

- Realización de una recopilación amplia de materiales, posters, folletos, etc. que incorporen los materiales publicitarios y de gestión realizados durante el tiempo de duración del proyecto. Incluyendo la selección, montaje en clip y organización del material más relevante para la exposición.

- Búsqueda, selección y organización de información preveniente de bibliotecas, instituciones como la cámara de comercio, consejería economía u OADE e Internet sobre los elementos fotografiados, para proceder al montaje en poster manual y poster para plotear mediante programa de edición y maquetación.

- Digitalización material, trasvase a www.biotopo.com, organización de la información a través de las herramientas php-nuke. Este será un proceso continuo durante el desarrollo del proyecto y será realizado por los alumnos de Transición a la Vida Adulta y Activa (Materia optativa de $4^{\circ}$ ESO).

- Realización de un video publicitario dela empresa. Publicación en INTERNET a través de un punto Windows Media.

- Dramatización y simulación de la actividad empresarial. 


\section{EVALUACIÓN}

La evaluación ha sido cualitativa, mediante cuestionario de preguntas abiertas, en dos fases para los profesores, también se ha tenido en cuenta la información vertida en las reuniones y en charlas informales. En el caso de los alumnos se ha utilizado la metodología del focus group en $4^{\circ}$ de la ESO en los grupos de Diversificación Curricular A y D para los alumnos. No pretendemos generalizar, sino mostrar una experiencia parcial que pueda servir para construir universalidades a través de su descripción. Cabe destacar algunas consideraciones:

- La motivación del profesorado ha sido alta, suponiendo un reto el no distanciarse del currículo oficial.

- La motivación de los alumnos ha sido buena y en algunos grupos extraordinaria, han existido excepciones y reticencias iniciales e incluso hubo una clase que no participó en el proyecto por decisión propia, ya que no se sintieron motivados.

- La proyección hacia fuera de nuestro centro ha sido buena, sin embargo algunos profesores indican que la exposición, dado el enorme trabajo realizado se ha visitado por debajo de sus expectativas.

- El poder participar en trabajos interdisciplinares e interdepartamentales ha sido valorado muy positivamente tanto por alumnos como por profesores.

- El profesorado tiene dificultades para reunirse y se culpabiliza en muchas ocasiones por no hacerlo. Hay que destacar la inexistencia de franjas horarias donde poder reunirse. Siempre habrá que hacerlo utilizando el tiempo libre o las tardes.

- El proceso de enseñanza aprendizaje ha sido visto como bueno o muy bueno tanto por los profesores como por los alumnos.

- Los alumnos han comprendido los objetivos, consideran que han aprendido, aunque algunos entienden que para ellos será difícil montar una empresa.

- Los alumnos se han concienciado en medio-ambiente, aunque muchos piensan que ya lo estaban.

- Los alumnos valoran positivamente el uso de Internet y de Biotopo.com

En general podemos decir que el nivel de satisfacción ha sido muy alto y que todos los estamentos del centro se han implicado de alguna manera en el mismo, hay que destacar el clima siempre caluroso y de armonía que ha reinado entre alumnos y profesores participantes. Desde el punto de vista académico, los profesores han manifestado que el proyecto les ha permitido desarrollar contenidos del currículo prescriptivo y que ha engarzado correctamente con el mismo en la mayoría de las materias. 
Ejemplos de nuestro trabajo con los alumnos podemos encontrarlos en:

Palabras presidenta Toxicodiver:

http://www.biotopo.com/pagina/toxicodiver/palabras.doc

Visita a Recytel

http://www.biotopo.com/pagina/toxicodiver/reciclaje/page1.htm

Anuncio televisivo Toxicodiver

mms://217.76.128.22/biocenosis.com/anuncioconfotoscompleto1.wmv

Tríptico exposición

http://www.elcorteingles.es/investigayexponlo/pdf/curs02004 05/FAtenea2.pdf

\section{REFERENCIAS BIBLIOGRÁFICAS}

Colás Bravo, M. P.; Buendía Eximan, L. (1998). Investigación Educativa. Madrid: Ediciones Alfar.

Dewey, J. (1966). Democracy and Education. New York: Free Press.

Elliot, J. (1988). Teachers as researchers: Implications for supervisions and teacher education, Nueva Orleans: American, Education Research Association (AERA).

Fernández Muñoz, R. (1998). El universo de las nuevas tecnologías: Información y nuevas tecnologías en la enseñanza. [en línea]. Disponible en: http://www. civila.com/universidades [consulta
2006, 7 de mayo]

Marqués, P. (1999). Evaluación de materiales multimedia. [en línea]. Disponible en: http://www.xtec. es/ pmarques/edusoft.htm [consulta 2006, 7 de mayo]

Martínez Bañuelos, J. J. (2002). Biocenosis.com, la naturaleza en la ciudad. Diseño, desarrollo, implementación y evaluación. Tesis doctoral. Madrid: UNED.

Martínez Bañuelos, J. J. (2006). La naturaleza en la ciudad. [en línea]. Disponible en: http://www.biotopo. com. [consulta 2006, 7 de mayo]

\section{PERFIL ACADÉMICO Y PROFESIONAL DEL AUTOR}

Julián José Martínez Bañuelos, Coordinador de Educación Ambiental del CNICE, en el proyecto Internet en el aula. Coordina el diseño instruccional de objetos digitales en transversales: en educación ambiental, bajo los auspicios de RED.ES. Jefe de estudios del IES Atenea de Alcalá de Henares, imparte clases en el Programa de Diversficación curricular. Es Biólogo y Doctor en Ciencias de la Educación.

E-mail: julian@biotopo.com 
DIRECCIÓN DEL AUTOR

\author{
IES ATENEA \\ C/ Río Arlanza nº 34 \\ Alcalá de Henares 28803 Madrid - España
}

Fecha de recepción del artículo: 23/10/06

Fecha de aceptación del artículo: 06/02/07 